\title{
Genetic algorithms for symbolic clustering
}

\author{
K CHIDANANDA GOWDA and T V RAVI \\ Department of Computer Science and Engineering, S J College of Engineering, \\ Mysore 570 006, India
}

MS received 18 March 1996

\begin{abstract}
This paper introduces a novel methodology for clustering of symbolic objects by making use of Genetic Algorithms (GAs). GAs are a family of computational models inspired by evolution. These algorithms encode potential solutions to specific problems on simple chromosome-like data structures and apply recombination operators to these structures so as to preserve critical information. A new type of representation for chromosome structure is presented here along with a new method for mutation. The efficacy of the proposed method is examined by application to numeric data of known number of classes and also to assertion type of symbolic objects drawn from the domain of fat oil, microcomputers, microprocessors and botany. The validity of the clusters obtained is examined.
\end{abstract}

Keywords. Symbolic clustering; symbolic similarity; symbolic dissimilarity; genetic algorithms; path length; spanning length; best spanning length.

\section{Introduction}

In conventional data analysis, objects are taken as numerical vectors. The clustering of such objects is achieved by minimizing intra cluster dissimilarity and maximizing inter cluster dissimilarity. A good survey of cluster analysis can be found in the literature (Duda \& Hart 1973; Diday \& Simon 1976; Bock 1987; Diday et al 1987; Jain \& Dubes 1988; Diday 1989).

Symbolic objects are extensions of classical data types. In conventional data sets, the objects are individualized, whereas in symbolic data sets, they are more unified by means of relationships. Based on the complexity, the symbolic objects can be of assertion, hoard or synthetic type. Some references to clustering of symbolic objects can be found in Diday (1990), Gowda \& Diday (1991a, 1992).

A symbolic clustering methodology is proposed in this paper, which makes use of GAs. An implementation of a genetic algorithm begins with a population of (typically random) chromosomes. One then evaluates these structures and allocates reproductive opportunities in such a way that chromosomes which represent a better solution to the target problem 


\author{
BEGIN /* genetic algorithm*/ \\ Generate initial population \\ Compute Fitness of each individual \\ WHILE NOT FINISHED Do \\ BEGIN /* Produce new generation */ \\ For Population Size Do \\ BEGIN /* Reproductive Cycle */ \\ Select two individuals from old generation \\ for mating /* biased in favour of fitter ones */ \\ Recombine the two individuals to give two offspring \\ Compute Fitness of the two offspring \\ Insert offspring in new generation \\ END \\ If population has converged THEN \\ finished $=$ TRUE \\ END \\ END.
}

Figure 1. A traditional genetic algorithm.

are given more chances to reproduce than those which are poorer solutions. The goodness of a solution is typically defined with respect to the current population. In a broader usage of the term, a genetic algorithm is any population-based model that uses selection and recombination operators to generate new sample points in a search space. The standard GA can be represented as shown in figure 1 .

\section{Proposed modified similarity and dissimilarity measures}

Similarity between $A$ and $B$ is written as,

$$
S(A, B)=S\left(A_{1}, B_{1}\right)+\cdots+S\left(A_{k}, B_{k}\right) .
$$

For the $k$ th feature, $S\left(A_{k}, B_{k}\right)$ is defined using the following three components:

(1) $S_{p}\left(A_{k}, B_{k}\right)$ due to position $p$,

(2) $S_{s}\left(A_{k}, B_{k}\right)$ due to span $s$,

(3) $S_{c}\left(A_{k}, B_{k}\right)$ due to content $c$.

Dissimilarity between $A$ and $B$ is written as,

$$
D(A, B)=D\left(A_{1}, B_{1}\right)+\cdots+D\left(A_{k}, B_{k}\right) .
$$

For the $k$ th feature, $D\left(A_{k}, B_{k}\right)$ is defined using the following three components:

(1) $D_{p}\left(A_{k}, B_{k}\right)$ due to position $p$,

(2) $D_{s}\left(A_{k}, B_{k}\right)$ due to span $s$,

(3) $D_{c}\left(A_{k}, B_{k}\right)$ due to content $c$. 


\subsection{Quantitative interval type of $A_{k}$ and $B_{k}$}

Let

$$
\begin{aligned}
& a l \text { and } a u \text { represent lower and upper limit of interval } A_{k} \text {, } \\
& b l \text { and } b u \text { represent lower and upper limit of interval } B_{k} \text {, } \\
& \text { inters }=\text { length of intersection of } A_{k} \text { and } B_{k} \text {, } \\
& l s=\text { span length of } A_{k} \text { and } B_{k}, \\
& =|\max (a u, b u)-\min (a l, b l)| \text {, }
\end{aligned}
$$

where $\max ()$ and $\min ()$ represent maximum and minimum values respectively. The similarity and dissimilarity between two samples $A_{k}$ and $B_{k}$ is defined on position and span. Similarity due to position is defined as

$$
S_{p}\left(A_{k}, B_{k}\right)=\sin \left[\left(1-\left((a l-b l) / u_{k}\right)\right) \times 90\right] .
$$

Similarity due to span is defined as

$$
S_{s}\left(A_{k}, B_{k}\right)=\sin [((l a+l b) /(2 \times l s)) \times 90],
$$

where $u_{k}$ denotes the length of the maximum interval of the $k$ th feature and

$$
\begin{aligned}
& l a=|a u-a l|, \\
& l b=|b u-b l| .
\end{aligned}
$$

Net similarity between $A_{k}$ and $B_{k}$ is

$$
S\left(A_{k}, B_{k}\right)=S_{p}\left(A_{k}, B_{k}\right)+S_{s}\left(A_{k}, B_{k}\right)
$$

Dissimilarity due to position is defined as

$$
D_{p}\left(A_{k}, B_{k}\right)=\cos \left[\left(1-\left((a l-b l) / u_{k}\right)\right) \times 90\right] .
$$

Dissimilarity due to span is defined as

$$
D_{s}\left(A_{k}, B_{k}\right)=\cos [((l a+l b) /(2 \times l s)) \times 90] .
$$

Net dissimilarity between $A_{k}$ and $B_{k}$ is

$$
D\left(A_{k}, B_{k}\right)=D_{p}\left(A_{k}, B_{k}\right)+D_{s}\left(A_{k}, B_{k}\right)
$$

\subsection{Qualitative type of $A_{k}$ and $B_{k}$}

For qualitative type of features the similarity component and dissimilarity component due to position are absent. The two components that contribute to similarity and dissimilarity are 
(1) span,

(2) content.

Let $l a=$ length of $A_{k}$ or number of elements in $A_{k}$,

$l b=$ length of $B_{k}$ or number of elements in $B_{k}$, inters $=$ number of elements common to $A_{k}$ and $B_{k}$,

$l s=$ span length of $A_{k}$ and $B_{k}$ combined, $=l a+l b-$ inters.

The similarity component due to span in defined as

$$
S_{s}\left(A_{k}, B_{k}\right)=\sin [(l a+l b) /(2 \times l s) \times 90] .
$$

The similarity component due to content is defined as

$$
\left.S_{c}\left(A_{k}, B_{k}\right)=\sin [\text { (inters } / l s) \times 90\right] .
$$

Net similarity between $A_{k}$ and $B_{k}$ is

$$
S\left(A_{k}, B_{k}\right)=S_{s}\left(A_{k}, B_{k}\right)+S_{c}\left(A_{k}, B_{k}\right) .
$$

The dissimilarity component due to span is defined as

$$
D_{s}\left(A_{k}, B_{k}\right)=\cos [((l a+l b) /(2 \times l s)) \times 90] .
$$

The dissimilarity component due to content is defined as,

$$
D_{c}\left(A_{k}, B_{k}\right)=\cos [(\text { inters } / l s) \times 90] .
$$

Net dissimilarity between $A_{k}$ and $B_{k}$ is

$$
D\left(A_{k}, B_{k}\right)=D_{s}\left(A_{k}, B_{k}\right)+D_{c}\left(A_{k}, B_{k}\right) .
$$

In conventional data analysis, whenever two samples that are merged are to be represented by a single sample, one of the frequently used methods is the mean of the two as a single representative. In symbolic data analysis, the concept of composite symbolic object (Gowda \& Diday 1991a, 1992) is used. A new method of forming a composite symbolic object is proposed in which

$$
\begin{aligned}
& a^{1} m=a m \times\left(n_{1} / n\right), \\
& b^{1} m=b m \times\left(n_{2} / n\right),
\end{aligned}
$$

where $a, b$ are the lowest and highest values considering $n$ samples, $n_{1}$ represents the number of samples between $a$ and $m, n_{2}$ represents the number of samples between $b$ and $m$.

\section{Methodology}

The problem here is to find the number of classes and class memberships in a data set of $N$ samples. In order to obtain the natural groups, the following method is proposed. The methodology makes use of the principle of mutation to obtain better solutions. Another important feature of the proposed method is that it makes use of a new type of representation for chromosome structure. 

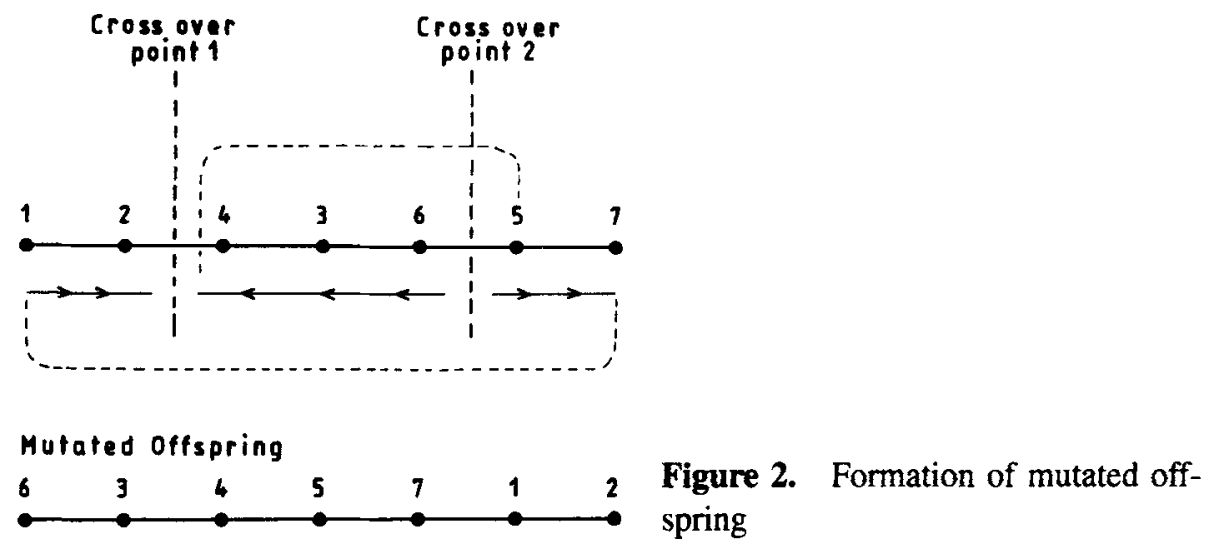

Stage 1: Generate $C$ number of random solutions. The solution would contain the samples connected in some order. If there are $N$ samples, randomly generate a number between 0 and $N$. Let it be $A_{0}$. Now generate one more number other than $A_{0}$. Let it be $B_{0}$. Connect $A_{0}$ and $B_{0}$. Next generate some number between 0 and $N$ other than $A_{0}$ and $B_{0}$. Let it be $C_{0}$. Connect $B_{0}$ and $C_{0}$. Repeat this until all the $N$ numbers are included. The solution would be in the form of a string (chromosome).

Compute the dissimilarities between $A_{0}$ and $B_{0}$ and hence find the path length. In the same way find the path length between $B_{0}$ and $C_{0}$ and so on. The sum of all the path lengths in the string would represent the spanning length.

Repeat the above procedure for $C$ solutions and hence find the strings and corresponding spanning lengths of each solution.

This would form the initial population.

Stage 2: Having $C$ random spanning lengths, our aim would be to obtain the best spanning length which has the least value. In order to obtain the best spanning length, we select an individual from the initial population and mutate it. The mutation is done in two phases.

Phase 1: The mutation is done by generating two crossover points and rearranging the samples in order to obtain a different string structure (chromosome). This is depicted in figure 2 .

Phase 2: The mutated offspring is further subjected to mutation as follows: Each sample in the string has two links connected to two different samples. Among the two links, the one that has the highest dissimilarity value is selected and replaced by a shorter link. This process is repeated for all the samples in the string.

Stage 3: The process of mutation, i.e. stage 2, is repeated till $90 \%$ of the initial population selected has the same spanning lengths.

Stage 4: Among the $C$ populations, the one that has the least spanning length is selected which would represent the best spanning length. This string structure would represent 
Table 1. Experimental results of randomly generated classes.

\begin{tabular}{lclc}
\hline $\begin{array}{l}\text { No. of } \\
\text { Gaussian } \\
\text { clusters } \\
\text { generated }\end{array}$ & $\begin{array}{c}\text { No. of } \\
\text { samples } \\
\text { generated }\end{array}$ & $\begin{array}{l}\text { Mean values } \\
\text { used for } \\
\text { generating } \\
\text { samples }\end{array}$ & $\begin{array}{c}\text { Classes } \\
\text { obtained by } \\
\text { proposed method }\end{array}$ \\
\hline 2 & 80 & 1,3 & 2 \\
3 & 120 & $1,3,5$ & 3 \\
4 & 160 & $1,3,5,7$ & 4 \\
5 & 200 & $1,3,5,7,9$ & 5 \\
6 & 240 & $1,3,5,7,9,11$ & 6 \\
7 & 280 & $1,3,5,7,9,11,13$ & 7 \\
8 & 320 & $1,3,5,7,9,11,13,15$ & 8 \\
\hline
\end{tabular}

the best solution to the given data. In order to obtain the classes, we adopt the following procedure.

(1) Compute the dissimilarites between each sample in the string, i.e. path lengths.

(2) Identify the inconsistent length, which is the path length having the highest value between two samples. Remove the inconsistent length to form two groups.

(3) Merge all the samples of each group and form two composite symbolic objects.

(4) Compute the similarity $S$ and dissimilarity $D$ between the two groups. If dissimilarity $D$ is greater than similarity $S$ then the inconsistent length is removed so as to result in two clusters or else the inconsistent length is placed back.

(5) Repeat steps 1 to 4 until no sample has dissimilarity greater than similarity.

\section{Results of simulation}

In order to corroborate the efficacy of the algorithm, several simulation studies were made, the results of which are given below. The clusterings obtained using the proposed method are examined for their validity using Hubert's T statistics (Jain \& Dubes 1988) approach. In order to compare the results, the validity of the clustering structures obtained by other methods is presented in table 11 below.

Table 2. Description of 2 classes of Iris data.

\begin{tabular}{lllll}
\hline Cluster no. & Feature 1 & Feature 2 & Feature 3 & Feature 4 \\
\hline 1 & $5.47-5.92$ & $2.55-2.73$ & $3.62-4.19$ & $1.13-1.31$ \\
2 & $4.83-4.86$ & $3.07-3.09$ & $1.38-1.43$ & $0.28-0.35$ \\
\hline
\end{tabular}




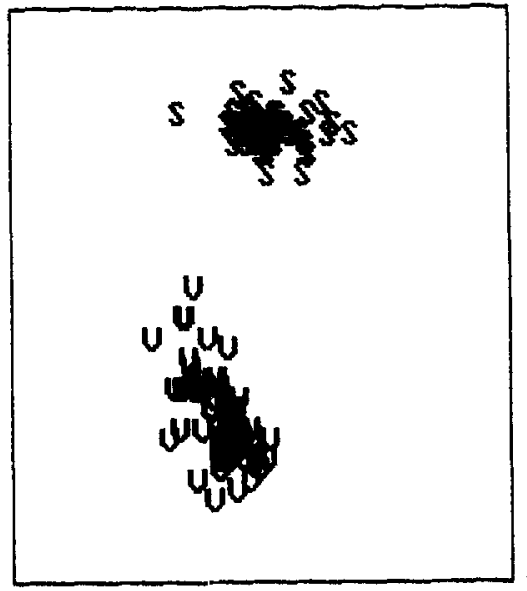

Figure 3. Plot of iris data; $x$-axis feature SW; $y$-axis - feature PL.

Example 1. The first example is such that the input data are of numeric type and the output data are symbolic. The objects of numeric type were drawn from a mixture of normal distributions with a known number of classes and classification so that the results show the efficacy of the algorithm. The test set was drawn from a mixture of $C$ normal distributions with mean $m_{i}$ and covariance matrix $C_{i}$ having individual variances of 0.15 and zero covariances. The different values of the number of classes and the means chosen are shown in table 1 and the test samples were independently generated using a Gaussian vector generator. The proposed method was used on this test data set. As indicated in table 1 , there is perfect agreement between the number of classes used for generating Gaussian clusters and the number of classes obtained by the proposed method. In all the seven cases, the classification results were in full agreement with the test samples generated.

Table 3. Fat oil data.

\begin{tabular}{lccccl}
\hline Sample & $\begin{array}{c}\text { Sp. gravity } \\
\left(\mathrm{g} / \mathrm{cm}^{3}\right)\end{array}$ & $\begin{array}{c}\text { m.p. } \\
\left({ }^{\circ} \mathrm{C}\right)\end{array}$ & Io. value & Sa. value & Fatty acids \\
\hline Linseed oil & $0.930-0.935$ & -27 to -8 & $170-204$ & $118-196$ & L,Ln,O,P,M \\
Perilla oil & $0.930-0.937$ & -5 to -4 & $192-208$ & $188-197$ & L,Ln,O,P,S \\
Cotton seed & $0.916-0.918$ & -6 to -1 & $94-113$ & $189-198$ & L,O,P,M,S \\
Sesame oil & $0.920-0.926$ & -6 to -4 & $104-116$ & $187-193$ & L,O,P,S,A \\
Camellia & $0.916-0.917$ & -21 to -15 & $80-82$ & $189-193$ & L,O \\
Olive oil & $0.914-0.919$ & 0 to 6 & $79-90$ & $187-196$ & L,O,P,S \\
Beef tallow & $0.860-0.870$ & 30 to 38 & $40-48$ & $190-199$ & O,P,M,S,C \\
Lard & $0.858-0.864$ & 22 to 32 & $53-77$ & $190-202$ & L,O,P,M,S,Lu \\
\hline
\end{tabular}

Abbreviations: Io - ionisation; sa - saponification; $\mathrm{L}$ - linoleic acid; $\mathrm{Ln}$ - linolenic acid; $\mathrm{O}$ - oleic acid; $\mathrm{P}$ - palmitic acid; $\mathrm{M}$ - myristic acid; $\mathrm{S}$ - stearic acid; $\mathrm{A}$ - arachic acid; $\mathrm{C}$ - capric acid; Lu - lauric acid 
Table 4. Description of classes in fat oil data.

\begin{tabular}{|c|c|c|c|c|c|c|}
\hline $\begin{array}{l}\text { Cluster } \\
\text { no. }\end{array}$ & Samples & $\begin{array}{c}\text { Sp. } \\
\text { gravity } \\
\left(\mathrm{g} / \mathrm{cm}^{3}\right)\end{array}$ & $\begin{array}{l}\left(0^{\circ} \mathrm{C}\right) \\
\text { m.p. }\end{array}$ & $\begin{array}{c}\text { Io. } \\
\text { value }\end{array}$ & $\begin{array}{c}\text { Sa. } \\
\text { value }\end{array}$ & $\begin{array}{l}\text { Fatty } \\
\text { acids }\end{array}$ \\
\hline 1 & $\begin{array}{l}0,1,2,3, \\
4,5\end{array}$ & $0.917-0.933$ & -7.75 to +3 & $191.25-196.75$ & $165.87-183.25$ & $\begin{array}{l}\text { L,Ln, } \\
\text { O,P, } \\
\text { M,S,A }\end{array}$ \\
\hline 2 & 6,7 & $0.862-0.864$ & $28.75-34.62$ & $49.75-60.25$ & $194.87-195.62$ & $\begin{array}{l}\text { L,O,P, } \\
\text { M,S, } \\
\text { Lu,C }\end{array}$ \\
\hline
\end{tabular}

Abbreviations: Io - ionisation; sa - saponification; $\mathrm{L}$ - linoleic acid; $\mathrm{Ln}$ - linolenic acid; $\mathrm{O}$ - oleic acid; $\mathbf{P}$ - palmitic acid; $\mathbf{M}$ - myristic acid; $\mathbf{S}$ - stearic acid; $\mathbf{A}$ - arachic acid; $\mathrm{C}$ - capric acid; Lu - lauric acid

Example 2. The example is so chosen so as to demonstrate the efficacy of the algorithm in clustering data belonging to two classes with many overlaps. The data set used is the well-known iris data set. This data set contains measurement of two species of iris. There are 50 patterns for each species and four features, namely petal length, petal width, sepal length and sepal width. The proposed method was applied to the two classes of iris data having 100 samples. The method resulted in two classes in perfect agreement with the data set considered. Two symbolic objects representing the two classes are shown in table 2, which also gives the descriptive information about the classes. The plot of the two classes obtained is as shown in figure 3 .

Table 5. Microcomputer data.

\begin{tabular}{llccll}
\hline Microcomputer & Display & $\begin{array}{c}\text { RAM } \\
(\mathrm{k})\end{array}$ & $\begin{array}{c}\text { ROM } \\
(\mathbf{k})\end{array}$ & MP & Keys \\
\hline Apple II & Colour TV & 48 & 10 & 6502 & 52 \\
Atari 800 & Colour TV & 48 & 10 & 6502 & $57-63$ \\
Commodore VIC 20 & Colour TV & 32 & $11-16$ & $6502 A$ & $64-73$ \\
Exidi Sorcerer & B\&W TV & 48 & 4 & Z80 & $57-63$ \\
Zenith H8 & Built-in & 64 & 1 & $8080 A$ & $64-73$ \\
Zenith H89 & Built-in & 64 & 8 & Z80 & $64-73$ \\
HP-85 & Built-in & 32 & 80 & HP & 92 \\
Horizon & Terminal & 64 & 8 & Z80 & $57-63$ \\
Ohio Sc Challenger & B\&W TV & 32 & 10 & 6502 & $53-56$ \\
Ohio Sc II Series & B\&W TV & 48 & 10 & $6502 C$ & $53-56$ \\
TRS-80 I & B\&W TV & 48 & 12 & Z80 & $53-56$ \\
TRS-80 III & Built-in & 48 & 14 & Z80 & $64-73$ \\
\hline
\end{tabular}


Table 6. Description of classes in microcomputer data.

\begin{tabular}{lclllll}
\hline $\begin{array}{l}\text { Cluster } \\
\text { no. }\end{array}$ & Samples & Display & $\begin{array}{l}\text { RAM } \\
(\mathbf{k})\end{array}$ & $\begin{array}{l}\text { ROM } \\
(\mathrm{k})\end{array}$ & MP & Keys \\
\hline 1 & $0,1,2,3,4,5$, & $\begin{array}{l}\text { Colour TV, } \\
\text { B \& W TV }\end{array}$ & 32,64 & $6-10$ & 6502 X & $55-73$ \\
& $7,8,9,10,11$ & & & & \\
& & $\begin{array}{l}\text { Terminal } \\
\text { Built-in } \\
\text { Built-in }\end{array}$ & 32 & 80 & HP & 92 \\
\hline
\end{tabular}

Table 7. Botanical data of 9 trees of 3 classes.

\begin{tabular}{lcc}
\hline $\begin{array}{l}\text { Class } 1 \\
\text { (Annonaceae) }\end{array}$ & $\begin{array}{c}\text { Class } 2 \\
\text { (Clasiaceae) }\end{array}$ & $\begin{array}{c}\text { Class } 3 \\
\text { (Mimosaceae) }\end{array}$ \\
\hline 0 degjknpCFR & 3 aehilmoswxzDFR & 6 begikmpBJKPR \\
1 dfhjknpCFR & 4 cfgilmoswxzDFR & 7 begiknpBJKPR \\
2 dehjknpCFR & 5 cegjlmoswxzDFR & 8 begjkmpBJKPR \\
\hline
\end{tabular}

Example 3. The data set for this example (table 3) is chosen from the domain of fats and oils (Ichino \& Yaguchi 1989) having four quantitative features of interval type and one nominal qualitative feature. The proposed method resulted in two classes. The samples of the classes were $\{0,1,2,3,4,5\}$ and $\{6,7\}$. Table 4 shows the two symbolic objects representing the two classes.

Example 4. The data set of microcomputers (table 5) (Ichino \& Yaguchi 1989) is considered for this experiment. The proposed method resulted in two classes. The samples of the classes were $\{0,1,2,3,4,5,7,8,9,10,11\}$ and 6 . Table 6 shows the description of the two classes.

Example 5. The data for this experiment (table 7) is considered from botany (Gowda \& Diday 1992). It consists of 9 trees belonging to 3 classes. The proposed method resulted in 3 classes. The samples of the classes were $\{0,1,2\},\{3,4,5\}$ and $\{6,7,8\}$. Table 8 shows the three symbolic objects representing the three classes.

Table 8. Description of classes in botanical data.

\begin{tabular}{llc}
\hline Cluster no. & \multicolumn{1}{c}{ Description } & Samples in cluster \\
\hline 1 & degjknphfCFR & $0,1,2$ \\
2 & cfgilmoswxzejahDFR & $3,4,5$ \\
3 & begjkmpinBJKPR & $6,7,8$ \\
\hline
\end{tabular}


Table 9. Microprocessor data.

\begin{tabular}{lccccl}
\hline MPU & Clock $(\mathrm{MHz})$ & Gen. reg* & Inst. (byte) & Cache size & Cache type \\
\hline i386DX & $16-33$ & 8 & 123 & - & Null \\
i386SX & $12-20$ & 8 & 123 & - & Null \\
i486DX & $25-50$ & 8 & 214 & 8192 & Common \\
i486SX & 20 & 8 & 129 & 8192 & Common \\
68020 & $12-33$ & 8 & 99 & 256 & Instruction \\
68030 & $16-50$ & 8 & 105 & 512 & Independent \\
68040 & 25 & 8 & 140 & 8192 & Independent \\
MB86901 & $20-25$ & 120 & 64 & - & Null \\
MB86930 & $20-40$ & 136 & 68 & 4096 & Independent \\
\hline
\end{tabular}

${ }^{*}$ General registers

Example 6. The data set of microprocessors (table 9) (Ichino \& Yaguchi 1989) is considered for this experiment. The proposed method resulted in two classes. The samples of the classes were $\{0,1,2,3,4,5,6,8\}$ and $\{7\}$. The description of the 2 classes is shown in table 10 .

As mentioned earlier, for purpose of comparison, results obtained by other methods are given in table 11 .

\section{Conclusion}

A new method for clustering symbolic objects is developed using GAs. A new type of representation for chromosome structure is presented along with two ways of mutating the chromosome structure. Several artificial and real-life data with known number of classes and classification assignments were used to establish the efficacy of the proposed methodology. Subsequently, the proposed methodology was applied to assertion type of symbolic data sets drawn from the domain of fat oil, microcomputers, botany, and microprocessors.

Table 10. Description of classes in microprocessor data.

\begin{tabular}{|c|c|c|c|c|c|c|}
\hline $\begin{array}{l}\text { Cluster } \\
\text { no. }\end{array}$ & Samples & $\begin{array}{l}\text { Clock } \\
(\mathrm{MHz})\end{array}$ & $\begin{array}{l}\text { Gen. } \\
\text { reg. }\end{array}$ & $\begin{array}{l}\text { Instructions } \\
\text { (byte) }\end{array}$ & $\begin{array}{l}\text { Cache } \\
\text { size }\end{array}$ & $\begin{array}{l}\text { Cache } \\
\text { type }\end{array}$ \\
\hline 1 & $\begin{array}{l}0,1,2,3,4 \\
5,6,8\end{array}$ & $22-28$ & 8 & $\begin{array}{l}123,99,140 \\
214,129 \\
105,68\end{array}$ & $\begin{array}{l}8192,256 \\
4096,512\end{array}$ & $\begin{array}{l}\text { Common, instruction, } \\
\text { Null, independent }\end{array}$ \\
\hline 2 & 7 & $20-25$ & 120 & 64 & - & Null \\
\hline
\end{tabular}


Table 11. Level of significance values for validating the clusters using Hubert's $T$ statistics.

\begin{tabular}{lcccc}
\hline Data & $\begin{array}{c}\text { Proposed } \\
\text { method }\end{array}$ & $\begin{array}{c}\text { Gowda \& Diday } \\
\text { (Using similarity) }\end{array}$ & $\begin{array}{c}\text { Gowda \& Diday } \\
\text { (Using dissimilarity) }\end{array}$ & Ichino \\
\hline Fat oil & 0.98 & 0.98 & 0.96 & 0.98 \\
Microcomputer & 0.90 & 0.67 & 0.67 & 0.90 \\
Microprocessor & 0.79 & - & - & 0.66 \\
Botanical & 1.00 & 1.00 & 1.00 & - \\
\hline
\end{tabular}

\section{References}

Bock H H (ed.) 1987 Classification and related methods of data analysis (Amsterdam: North Holland)

Diday E (ed.) 1989 Data analysis, learning symbolic and numeric knowledge (Antibes, France: Nova Science)

Diday E 1990 From numerical to symbolic clustering, COMPSTAT Dubrovnik, Yugoslavia

Diday E, Simon J C 1976 Clustering analysis: Communication and cybernetics (New York: Springer Verlag) vol.10, pp 47-92

Diday E, Hayashi C, Jambu M, Ohsumi N (eds) 1987 Recent developments in clustering and data analysis (New York: Academic Press)

Duda R O, Hart PE 1973 Pattern classification and scene analysis (New York: Wiley Interscience)

Gowda K C, Diday E 1991a Symbolic clustering using a new dissimilarity measure. Pattern Recogn. 24: 567-578

Gowda K C, Diday E 1991b Unsupervised learning through symbolic clustering. Pattern Recogn. Lett. 12: 259-264

Gowda K C, Diday E 1992 Symbolic clustering using a new similarity measure. IEEE Trans. Syst. Man Cybern. 22: 368-378

Ichino M 1988 General metrics for mixed features - The Cartesian space theory for pattern recognition. Proc. IEEE Conference on Systems, Man and Cybernetics

Ichino M, Yaguchi H 1989 Generalised Minkoswki metrics for mixed features. Trans. IEICE Japan J 72-A: 398-405 (in Japanese)

Jain A K, Dubes R C 1988 Algorithms for clustering data (Englewood Cliffs, NJ: Prentice Hall) 